\title{
Impulsive Personality Predicts Dopamine-Dependent Changes in Frontostriatal Activity during Component Processes of Working Memory
}

\author{
Roshan Cools, ${ }^{1,2}$ Margaret Sheridan, ${ }^{1}$ Emily Jacobs, ${ }^{1}$ and Mark D’Esposito ${ }^{1}$ \\ ${ }^{1}$ Helen Wills Neuroscience Institute, University of California, Berkeley, Berkeley, California 94720-3190, and ${ }^{2}$ Behavioural and Clinical Neuroscience \\ Institute, University of Cambridge, Cambridge CB2 3EB, United Kingdom
}

\begin{abstract}
Dopaminergic drugs affect a variety of cognitive processes, but the direction and extent of effects vary across individuals and tasks. Paradoxical effects are observed, by which the same drug causes cognitive enhancing as well as adverse effects. Here, we demonstrate that individual differences in impulsive personality account for the contrasting effects of dopaminergic drugs on working memory and associated frontostriatal activity. We observed that the dopamine $\mathrm{D}_{2}$ receptor agonist bromocriptine improved the flexible updating (switching) of relevant information in working memory in high-impulsive subjects, but not in low-impulsive subjects. These behavioral effects in high-impulsive subjects accompanied dissociable effects on frontostriatal activity. Bromocriptine modulated the striatum during switching but not during distraction from relevant information in working memory. Conversely, the lateral frontal cortex was modulated by bromocriptine during distraction but not during switching. The present results provide a key link between dopamine $\mathrm{D}_{2}$ receptor function, impulsivity, and frontostriatal activity during component processes of working memory.
\end{abstract}

Key words: dopamine; working memory; prefrontal cortex; basal ganglia; fMRI; impulsivity

\section{Introduction}

The mesocorticolimbic dopamine (DA) system is implicated in working memory (Brozoski et al., 1979; Sawaguchi and Goldman-Rakic, 1991). However, the effects of dopaminergic drugs are complex and depend on task demands and associated neural systems (Cools and Robbins, 2004). Recent work suggests that DA may have opposite effects depending on the neural site of modulation [prefrontal cortex (PFC) vs striatum] (Crofts et al., 2001; Frank et al., 2001; Gruber et al., 2006). DA in the PFC is thought to stabilize representations by reducing susceptibility to distraction (Durstewitz et al., 2000; Seamans and Yang, 2004). Conversely, DA in the striatum may rapidly update representations in a task-relevant manner (Frank et al., 2001; Gruber et al., 2006). In the current study, we aimed to test the hypothesis that DA modulates the striatum and frontal cortex during the flexible updating and stable maintenance of representations, respectively, by examining the effects of the $\mathrm{D}_{2}$ receptor agonist bromocriptine with functional magnetic resonance imaging (fMRI).

Dopaminergic drug effects vary not only across task demands but also across individuals (Cools and Robbins, 2004). Paradoxical effects are observed, by which drugs improve performance in

Received Feb. 11, 2007; revised April 17, 2007; accepted April 18, 2007

This work was supported by National Institutes of Health Grants MH63901, NS40813, and DA02060 and the Veterans Administration Research Service. We are grateful to Lee Altamirano and Elizabeth Kelley for assistance with data acquisition and analysis.

Correspondence should be addressed to Roshan Cools, Behavioural and Clinical Neuroscience Institute, Department of Experimental Psychology, University of Cambridge, Downing Street, Cambridge CB2 3EB, UK. E-mail: roshan.cools@gmail.com.

D0I:10.1523/JNEUROSCI.0601-07.2007

Copyright $\odot 2007$ Society for Neuroscience $\quad$ 0270-6474/07/275506-09\$15.00/0 individuals with suboptimal DA and poor performance but impair performance in individuals with already optimized DA and good performance (Kimberg et al., 1997; Arnsten, 1998; Mattay et al., 2003; Phillips et al., 2004). Here, we demonstrate that dopaminergic drug effects can also be predicted from trait impulsivity. We observed differential effects of bromocriptine in highand low-impulsive subjects, who were preselected based on selfreport trait impulsivity. The rationale for impulsivity-based subject selection was threefold. First, impulsivity is the primary trait thought to mediate vulnerability to impulse-control disorders, such as drug addiction (Dawe and Loxton, 2004), and is associated with enhanced sensitivity to dopaminergic drug effects (Boileau et al., 2006). Second, trait impulsivity is associated with low baseline $\mathrm{D}_{2} / \mathrm{D}_{3}$ receptor binding (Dalley et al., 2007). Third, impulsive disorders are characterized by working memory impairment and abnormalities in frontostriatal circuits (Jentsch and Taylor, 1999; Schweitzer et al., 2000; Castellanos and Tannock, 2002; Willcutt et al., 2005), and impulsive personality correlates with low baseline cognitive performance (Keilp et al., 2005). Accordingly, we stratified our effects by trait impulsivity and predicted that high-impulsive individuals would be particularly sensitive to the effects of bromocriptine on working memory.

In keeping with this prediction is recent evidence that dopaminergic medication in patients with attention-deficit/hyperactivity disorder (ADHD) remediates difficulty with the flexible updating and stable maintenance of task-relevant information in working memory (Frank et al., 2007). Our study extends this work by assessing drug effects on striatal and frontal activity during these component processes in working memory in high- and 


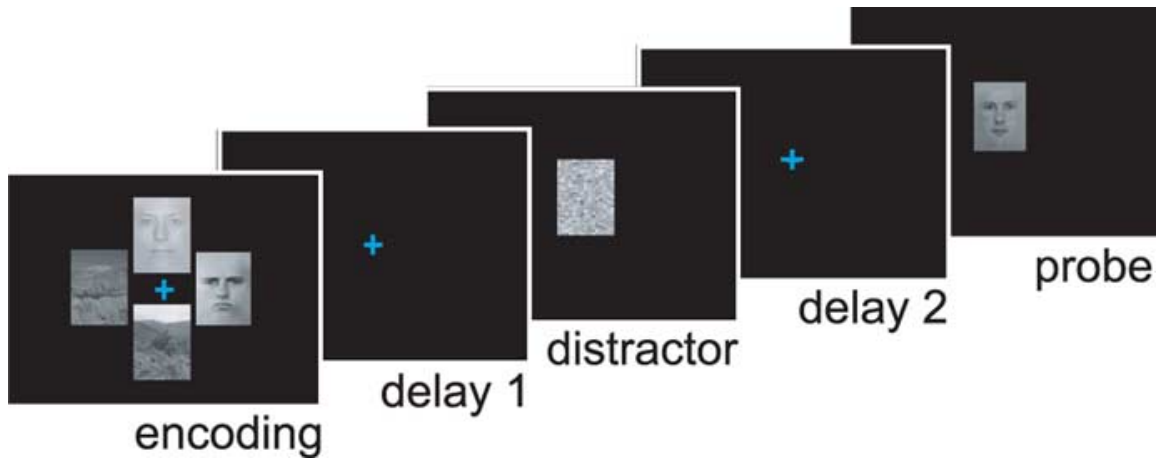

Figure 1. Example trial from the experimental paradigm. In this trial, a blue fixation cross instructed subjects to attend to the faces and ignore the scenes. The encoding period (1000 ms) was followed by an $8000 \mathrm{~ms}$ delay period, after which a scrambled distractor was presented. After a second delay $(8000 \mathrm{~ms})$, subjects were probed to make a left/right button press depending on whether or not the probe face matched one of the two encoding faces.

low-impulsive subjects. Subjects performed a delayed match-tosample paradigm that enabled the separate assessment of switching and resistance to distraction in working memory. We predicted that bromocriptine would improve switching and resistance to distraction in high-impulsive subjects but that it would impair these processes in low-impulsive subjects. Moreover, we predicted that these effects would be accompanied by modulation of the striatum and frontal cortex, respectively.

\section{Materials and Methods \\ Subjects}

Twenty-eight healthy right-handed volunteers participated in this experiment. The study was approved by the University of California, Berkeley Committee for the Protection of Human Subjects and performed in accordance with the Declaration of Helsinki. All volunteers gave written informed consent and were paid for participation ( $\$ 250$ after completion).

A large sample $(n=1118)$ of young college undergraduates were prescreened on the self-report Barratt Impulsiveness Scale (BIS-11) (Patton et al., 1995) as part of the Research Participation Program organized by the Haas School of Business at the University of California, Berkeley. We selected two groups of subjects from the tail ends (i.e., the top and bottom 15th percentiles) of the normal distribution of total BIS scores (mean score, 64.4; median score, 64; range, 39-99; SD, 9.7). Fourteen healthy high-impulsive subjects were recruited from the top 15th percentile ( $n=163$; scores $\geq 75$; mean score, 80.0 ; median score, 79 ; SD, 4.8). Similarly, 14 low-impulsive subjects were recruited from the bottom 15 th percentile $(n=168$; scores $\leq 54$; mean score, 50.1 ; median score, 51 ; $\mathrm{SD}, 3.3)$. Six subjects had to be excluded because of excessive movement and other reasons (supplemental material, available at www.jneurosci.org). Data are reported from 12 low-impulsive subjects (mean age \pm $\mathrm{SD}, 19.6 \pm 1.6$; range, $18-22$ ) and 10 high-impulsive subjects (mean age $\pm S D, 20.8 \pm 2.1$; range, 18-26).

Exclusion criteria were an episode of loss of consciousness, use of psychotropic drugs, sleeping pills, heavy marijuana use ( $>10$ times), and any history of medical, neurological, or psychiatric disorder.

\section{General procedure}

All subjects were invited to visit the Helen Wills Neuroscience Institute on three occasions: on the first occasion, which lasted $\sim 2.5 \mathrm{~h}$, they were interviewed for suitability, administered background neuropsychological tests, and explained the procedure. On the second and third occasions, subjects were scanned, after intake of a lactose placebo or an oral dose of the $\mathrm{DA} \mathrm{D}_{2}$ receptor agonist bromocriptine $[1.25 \mathrm{mg}$, a dose commonly used in psychopharmacological studies and well tolerated by subjects (Gibbs and D'Esposito, 2005a,b)], according to a double-blind, placebo-controlled crossover design.

After arrival at the institute [time 0 (T0)], subjects performed their first minibattery (described below), and their blood pressure was taken.
Twenty minutes after arrival (T20), subjects ingested a placebo or a bromocriptine capsule, with a glass of soymilk. The minibatteries were repeated at T50 and T80, after which the subjects were prepared for their scan and taken to the scanner. They entered the scanner at T110 (90 min after drug intake). After completion of the scan, subjects were administered a fourth minibattery (at $\sim \mathrm{T} 225$ ), and their blood pressure was measured again. Subjects were guided back to the testing room, where they completed a series of neuropsychological tests (results reported elsewhere). The testing session ended with a final minibattery at $\sim \mathrm{T} 335$.

Subjects were instructed to avoid eating large meals on the day of and before their visit but to have a light meal $\sim 1 \mathrm{~h}$ before arrival. They were asked to refrain from caffeine and cigarettes on the days of the scan. Light snacks were provided after drug intake and after scanning, before the final neuropsychological testing session.

\section{Background neuropsychology}

Neuropsychological profile at baseline. During the preliminary testing session, subjects completed a neuropsychological battery, which included (1) the Daneman and Carpenter reading span (Daneman and Carpenter, 1980) (supplemental material, available at www.jneurosci.org); (2) the North American Adult Reading Test (Nelson, 1982); (3) the Beck Depression Inventory (Beck et al., 1961); (4) the forward digit span; (5) the forward and backward spatial span (Corsi Block-Tapping test) (Milner, 1971); (6) the Wisconsin Card Sorting Test (Grant and Berg, 1948); (7) a paper-and-pen version of the Stroop task (Stroop, 1935); (8) letter and semantic fluency tasks (Benton, 1968); (9) a six-trial practice block of the experimental task to be performed in the scanner; and (10) the Montreal Cognitive Assessment (Nasreddine et al., 2005) (to assess mild cognitive impairment; analogous to the Mini Mental State Examination).

Performance on background cognitive tests on the scanning days. On each scanning day, subjects performed a minibattery on five occasions. This minibattery included the backwards digit span, visual analog scales (anxious, happy, sad, nauseous, drowsy, jittery, fatigue, and dizzy) and a motor tapping test.

\section{Experimental design}

During scanning, subjects performed a delayed match-to-sample task, which consisted of four blocks with 32 trials per block ( $\sim 13 \mathrm{~min} / \mathrm{block})$ Before entering the scanner, subjects were reminded of the instructions by referring to the six-trial practice session they had performed on the preliminary testing session.

On each trial, subjects were presented four pictures: two novel faces and two novel scenes, which were arranged around a colored fixation cross (location randomized). They had to encode, maintain, and retrieve either the faces or the scenes, depending on the color of the fixation cross, which thus served as an instruction cue. Subjects were instructed to memorize the faces when the fixation cross was blue and to memorize the scenes when the cross was green. The encoding period (duration, 1000 $\mathrm{ms}$ ) was followed by an $8000 \mathrm{~ms}$ delay period during which the stimuli were removed from the screen, and only the colored fixation cross was presented. After this first delay period, a distractor was presented (duration, $2000 \mathrm{~ms}$ ), which subjects were instructed to ignore. This distractor allowed us to test the hypothesis that DA-dependent frontal mechanisms are involved in maintaining relevant representations despite similar, but irrelevant, stimuli. The distractor was followed by a second delay period (duration, $8000 \mathrm{~ms}$; colored fixation cross present), after which subjects were probed to respond with the right or left finger depending on whether or not the probe matched one of the two task-relevant encoding stimuli (Fig. 1).

The order of blue-face and green-scene trials was pseudorandomized and unpredictable, enabling the measurement of switching (from attending to faces to attending to scenes and vice versa) against a background of 
nonswitching (face to face or scene to scene; the minimum/maximum number of consecutive nonswitch trials was $1 / 3$ ). We also manipulated the type of distractor that subjects encountered during the delay. On some trials, subjects viewed a scrambled face or scene; on other trials, the distractor was a nonscrambled, novel face or scene. The stimulus modality of this nonscrambled distractor (face or scene) was always congruent with the task-relevant stimulus modality. Based on previous work from our group (Yoon et al., 2006), we predicted that the performance at probe would be impaired after the congruent novel distractor relative to the scrambled distractor. Consistent with previous and predicted findings, pilot data from 16 young healthy volunteers had indeed revealed slower responses at probe after the nonscrambled than the scrambled distractor, for faces $\left(F_{(1,15)}=2.2 ; p=0.04\right)$. Moreover, reaction times were significantly prolonged on switch trials compared with RTs on nonswitch trials, regardless of stimulus modality (i.e., faces or scenes; $F_{(1,15)}=19.1$; $p=0.001)$. The effect of face distraction was replicated in 13 older healthy volunteers $\left(F_{(1,12)}=7.4 ; p=0.02\right)$, although an adaptation of the paradigm for testing in patients rendered the task insensitive to switching in that study (data reported elsewhere).

Distractors on switch trials were always nonscrambled, so that, in all, there were three trial types: (1) switch trials with nonscrambled distractors $(n=56),(2)$ nonswitch trials with nonscrambled distractors $(n=$ 36 ), and (3) nonswitch trials with scrambled distractors $(n=36)$. Stimulus modality and match/nonmatch status were counterbalanced between these trial types. There were two critical performance measures. The calculation of the switch cost was restricted to the nonscrambled distractor trials and performed by subtracting error rates and reaction times (RTs) at probe on nonswitch trials from error rates and RTs at probe on switch trials. The calculation of the distractor cost was restricted to nonswitch trials and performed by subtracting performance after scrambled distractors from that after nonscrambled distractors.

\section{Behavioral analyses}

Accuracy and RT data were submitted to two repeated-measures ANOVAs. The first ANOVA addressed the effects on attentional switching and included the between-subjects factor group and the within-subject factors drug and switch. The second ANOVA addressed the effects on distraction, and the within-subject factor distractor replaced the factor switch. The behavioral analysis included the two high-impulsive subjects who were excluded from the fMRI analysis because of excessive movement. Exclusion of these subjects did not change the results. Data from the bromocriptine session of one high-impulsive subject was missing because of equipment (e-prime-recording) failure.

\section{Image acquisition}

Imaging data were collected using a Varian (Palo Alto, CA) INOVA 4T scanner equipped with a transverse electromagnetic send-and-receive radio frequency head coil. Functional data were obtained using a twoshot $\mathrm{T} 2{ }^{\star}$-weighted echo-planar imaging sequence sensitive to blood oxygenation level-dependent contrast (repetition time, $2000 \mathrm{~ms}$; echo time, $28 \mathrm{~ms}$; field of view, $22.4 \mathrm{~cm}^{2}$; matrix size, $64 \times 64$; in-plane resolution, $3.5 \times 3.5 \mathrm{~mm}$ ). Each functional volume consisted of 185 -mm-thick axial-oblique slices separated by a $0.5 \mathrm{~mm}$ interslice gap and provided nearly whole-brain coverage. Two T1-weighted anatomical scans were also acquired [a gradient-echo multislice and a magnetization-prepared fast low-angle shot (MP-FLASH) three-dimensional sequence].

\section{Image analyses}

After acquisition, MRI data were converted to Analyze format, corrected for slice acquisition time and interpolated to $1 \mathrm{~s}$ temporal resolution (one-half of the total repetition time). Subsequent processing was performed using SPM2 (Wellcome Department of Cognitive Neurology, London, UK; http://www.fil.ion.ucl.ac.uk) run under Matlab6.5 (The MathWorks, Natick, MA). Functional data were realigned to the first volume acquired, spatially normalized, and spatially smoothed using a Gaussian kernel (10 mm full-width at half-maximum). For spatial normalization, the individual subject's MP-FLASH was skull stripped [using the brain extraction tool (Smith, 2002)], coregistered to the mean functional image, and subsequently normalized to the Montreal Neurological Institute (MNI) skull-stripped structural template. The obtained nor-

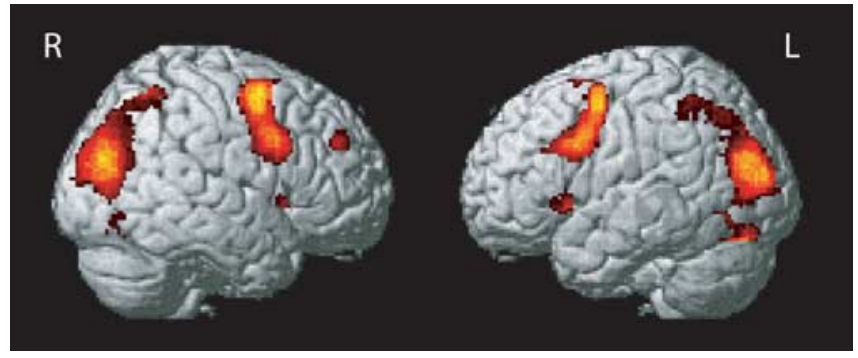

Figure 2. Encoding-related suprathreshold clusters ( $p_{\mathrm{FWE}}<0.00001$ ). Left, Right hemisphere (R); right, left hemisphere (L). Note that the small clusters that appear to be located in the posterior/inferior portions of the inferior frontal gyrus were in fact located in the insulae/ superior temporal gyri.

malization parameter set was then written to the functional images. Time series were high-pass filtered (128 s cutoff).

A canonical hemodynamic response function was used as a covariate in a general linear model. The hemodynamic response function was modeled to the onset of each event with independent regressors for each stage and each trial type (supplemental material, available at www. jneurosci.org). We concentrate on the following contrasts of interest: (1) encoding-related activity during switch trials minus encoding-related activity during nonswitch trials and (2) distractor-related activity during nonscrambled stimuli minus distractor-related activity during scrambled, novel stimuli (collapsed across stimulus modality). We collapsed data across correct and incorrect trials, because supplementary analyses revealed that our effects of interest (of group, drug, and group $\times$ drug) did not differ between correct and incorrect trials (either as a function of switching or distraction). Inclusion of both correct and incorrect trials considerably enhanced the statistical power of the analyses.

To calculate whole-brain maps for the critical three-way group $\times$ drug $\times$ trial type interactions, each individual's placebo session's contrast was subtracted from the corresponding bromocriptine session's contrast. These difference contrasts were taken to a second-level group two-sample $t$ test, allowing a direct comparison between the drug effects in high-impulsive subjects with those in low-impulsive subjects. Data from whole-brain maps are presented in Figures 5 and 7.

\section{Regions of interest}

The specific predictions allowed us to concentrate on region of interest (ROI) analyses. The striatal ROIs were selected directly from the Automated Anatomical Labeling interface with SPM, which was developed by Tzourio-Mazoyer et al. (2002), based on an anatomical parcellation of the spatially normalized single-subject high-resolution T1 volume provided by the MNI. From this interface, we selected the right and left putamen and the right and left caudate nucleus for ROI analysis. Although there is considerable agreement about the anatomical boundaries of and within striatal regions, a clear definition of both anatomical and functional subregions within the lateral frontal cortex is lacking. Therefore, lateral frontal ROIs were derived from the data themselves. To obtain task-related frontal activation clusters that were orthogonal to our comparisons of interest (between drug session and groups), contrasts depicting encoding-related activity were calculated across the two treatment sessions and across all subjects $(n=22)$. We chose to select frontal ROIs from the encoding contrast (reflecting activity correlating with the mean of all encoding regressors vs the mean of all other regressors) (Fig. 2), because this was hypothesized to be the most sensitive contrast for detecting changes as a function of switching (which occurred during the encoding period). This encoding contrast revealed activation in large parts of the posterior and frontal cortex.

A thresholded map (at $p_{\mathrm{FWE}}<0.00001$; this threshold was the lowest threshold to reveal discrete clusters in the frontal cortex) revealed three discrete lateral frontal clusters in (1) the right middle frontal gyrus (MFG); (2) the superior and posterior portion of the right inferior frontal gyrus (IFG), extending into the right precentral gyrus (PCG) and the rostral cingulate zone; and (3) the superior and posterior portion of the 
Table 1. Encoding-related suprathreshold clusters at Talairach $y>0\left(p_{\mathrm{FWE}}<\right.$ 0.00001)

\begin{tabular}{lllc}
\hline Label & $\begin{array}{l}\text { Talairach coordinates }(x, y, z) \\
\text { of peak locus }\end{array}$ & $t$ & Cluster size (voxels) \\
\hline Right IFG/PCG & $46,8,32$ & 16.65 & 3967 \\
Left IFG/PCG & $-46,0,54$ & 13.47 & 1528 \\
Right MFG & $36,46,32$ & 10.06 & 76 \\
\hline
\end{tabular}

left IFG, extending into the left PCG (for peak loci, cluster sizes, and $t$ values, see Fig. 2, Table 1).

To select discrete lateral frontal clusters that did not extend into the PCG or the medial PFC, we masked the encoding map with the right and left IFG, as defined by Tzourio-Mazoyer et al. (2002). This resulted in three ROIs: the right IFG, the left IFG, and the right MFG.

The statistical models described above were reapplied to the average signal within the ROIs for each subject's session, using the MarsBar tool for SPM2 (Brett et al., 2002). Data from each of these ROIs were extracted and transformed into percentage signal change (for details, see http:// marsbar.sourceforge.net/faq.html\#percent_signal). These data were submitted to two types of repeated-measures ANOVAs (in SPSS 11.0; SPSS, Chicago, IL). The first type of ANOVA addressed our primary hypothesis that bromocriptine has different effects on switch-related activity in the striatum depending on trait impulsivity. In particular, we predicted that the drug would modulate activity in the putamen during switching. This hypothesis was based on our previous work in patients with Parkinson's disease (who suffer DA depletion primarily in the putamen) (Cools et al., 2001a,b, 2003) and patients with focal lesions in the putamen (Cools et al., 2006) and an fMRI study that revealed putamen activity during switching (Cools et al., 2004). Thus, the first analyses were performed on switch-related activity in each of the striatal ROIs separately (right/left putamen and right/left caudate nucleus) and included the between-subjects factor group and the within-subject factor drug. Switch-related activity was calculated by subtracting encoding-related activity on nonswitch trials from encoding-related activity on switch trials. The second set of ANOVAs tested our second hypothesis, that these effects on striatal activity during switching differed from effects on lateral frontal activity during distraction. For these analyses, we collapsed the four sub-ROIs in the striatum (to render the second set of analyses orthogonal to the first analysis set and to approximately match the size of the striatal and frontal ROIs) and compared activity in this striatal metaROI with a meta-ROI in the lateral frontal cortex (data collapsed across the three frontal sub-ROIs described above). It was performed with group as the between-subjects factor and ROI (striatum vs lateral frontal cortex), process (switch-related activity vs distractor-related activity), and drug as within-subject factors. Supplementary analyses explored effects in each lateral frontal cortex ROI separately. Finally, we report results from the striatum (the putamen and caudate nucleus) and the lateral frontal cortex [Talairach coordinates, $y>0$ and $x>+16$ or $x<$ -16 , with the $x$-coordinates based on Owen et al. (1999)] as revealed by whole-brain analyses of the critical group by drug $\times$ trial type interactions (thresholded at $p=0.001$, uncorrected for multiple comparisons). Other regions of no interest, for which we had no a priori predictions, were explored at a higher threshold of $p=0.05$, corrected for multiple comparisons according to the familywise error rate.

\section{Results}

\section{Background neuropsychology}

The drug effects that we report below were not attributable to nonspecific effects on global cognitive or motor function. There were no drug effects on our background neuropsychological tests (see supplemental material, available at www.jneurosci.org) other than a significant effect of drug on motor tapping speed in both the high- and the low-impulsive subjects (drug $\times$ time interaction, $\left.F_{(1,20)}=5.0 ; p=0.04\right)$. This profile enabled us to evaluate the drug effects of interest on working memory against a

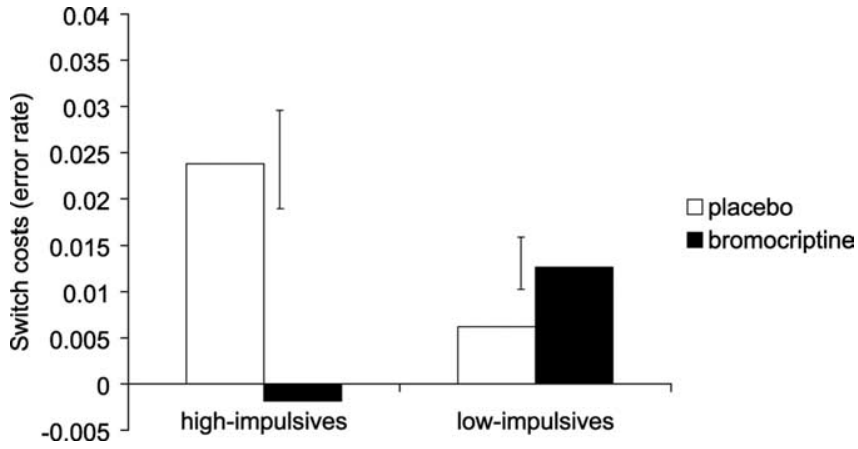

Figure 3. Behavioral switch costs as a function of trait impulsivity and drug. Switch costs represent the mean proportion of errors on switch trials minus the mean proportion of errors on nonswitch trials. Error bars represent SEs of the difference between treatment sessions.

background of unaltered basic cognitive abilities and subjective effects.

As would be expected, the high-impulsive subjects performed more poorly than the low-impulsive subjects on the interference condition of the Stroop test $\left(F_{(1,20)}=7.8 ; p=0.01\right)$. In addition, they had a significantly lower reading span, as measured with the listening version of the Daneman and Carpenter Read Span test $\left(F_{(1,20)}=5.4 ; p=0.03\right)$.

\section{Performance effects}

We first investigated performance effects (measured at probe) of bromocriptine as a function of switching with an ANOVA with the between-subjects factor group and the within-subject factors drug and switch (switch vs nonswitch). In Figure 3, we present the mean error switch costs for the high- and low-impulsive subjects separately as a function of drug (for data as a function of trial type, see Table 2). The drug effect on switching depended on trait impulsivity, as indicated by a significant group $\times$ drug $X$ switch interaction $\left(F_{(1,21)}=7.1 ; p=0.01\right)$. This three-way interaction explained why the main effect of switching did not reach significance $\left(F_{(1,21)}=2.7 ; p=0.1\right)$ and indicates that switching in the present paradigm depended on both trait impulsivity and drug session.

These observations were confirmed by additional statistical analyses. Although the switch cost at baseline did not differ significantly between the two groups $\left(F_{(1,21)}=1.3\right)$, bromocriptine had different effects on switching in the high- and low-impulsive subjects. Thus, bromocriptine significantly attenuated the switch cost in high-impulsive subjects $\left(F_{(1,10)}=5.5 ; p=0.04\right)$ but did not affect the switch cost in low-impulsive subjects $\left(F_{(1,11)}=1.3\right.$; $p=0.3)$. Simple interaction effects analyses confirmed that the high-impulsive subjects exhibited significantly more errors on switch trials than on nonswitch trials, when tested on placebo $\left(F_{(1,11)}=5.1 ; p=0.05\right)$. Bromocriptine abolished this switch cost $\left(F_{(1,10)}=0.04 ; p=0.8\right)$. Although inspection of Table 2 reveals that bromocriptine altered performance primarily on switch trials but not on nonswitch trials, the effects of bromocriptine did not reach significance when switch and nonswitch trials were analyzed separately $(p>0.2)$.

There was no significant distractor cost (error rate after the nonscrambled distractor minus error rate after the scrambled distractor) across all sessions and subjects $\left(F_{(1,21)}=0.4\right)$, and there were no effects of group or drug as a function of distraction (group $\times$ drug $\times$ distractor, $F_{(1,21)}=0.4$; drug $\times$ distractor, $F_{(1,21)}=0.2$; group $\times$ distractor, $\left.F_{(1,21)}=0.9\right)$.

No drug or group effects were found on RTs (group $\times$ drug $\times$ 
switch, $F_{(1,21)}=1.5, p=0.2$; drug $\times$ switch, $F_{(1,21)}=0.4, p=0.5$; group $\times$ drug $\times$ distractor, $F_{(1,21)}=0.07, p=0.8$; drug $\times$ distractor, $\left.F_{(1,21)}=0.3, p=0.6\right)$. There was no main effect of switching $\left(F_{(1,21)}=1.0\right)$ or distraction $\left(F_{(1,21)}=0.8\right)$ in terms of RT, even if face- and scenerelevant trials were analyzed separately from the placebo session only (switching faces, $F_{(1,21)}=0.3$; switching scenes, $F_{(1,21)}$ $=0.2$; distraction faces, $F_{(1,21)}=0.8$; distraction scenes, $\left.F_{(1,21)}=0.6\right)$. This contrasted with previous pilot data from young and older volunteers who showed a significant RT switch cost as well as a significant RT distractor cost (see Materials and Methods). This difference between the previous behavioral study and the present fMRI study may reflect adoption of different performance strategy in the scanner environment. Indeed, the mean RT in the previous study was much faster $(1017 \mathrm{~ms}$; SEM, $74 \mathrm{~ms}$ ) than the mean RT in the current study (1273.8 ms; SEM, $63 \mathrm{~ms})$. Thus, it is possible that the predicted RT effects did not surface because subjects did not sufficiently emphasize speed. Perhaps the longer RTs and long delays enabled them to retrieve information from long-term memory, circumventing distractordisrupted working memory.

In sum, the behavioral effects of bromocriptine depended on trait impulsivity. The drug improved performance on switch trials relative to nonswitch trials in the high-impulsive subjects but not in the low-impulsive subjects. There were no effects as a function of distraction.

\section{Neural activity in the striatum}

First we conducted hypothesis-driven ROI analyses in a priori defined ROIs to test whether the drug modulated activity in specific subregions of the striatum during switching. In particular, we predicted that the drug would modulate activity in the putamen during switching. This hypothesis was based on our previous work in patients with Parkinson's disease [who suffer DA depletion primarily in the putamen (Cools et al., 2001a,b, 2003)] and patients with focal lesions in the putamen (Cools et al., 2006) and an fMRI study that revealed putamen activity during switching (Cools et al., 2004). In keeping with this a priori hypothesis, we found that the performance effects on switching were paralleled by effects on activity in the putamen during switching. In Figure 4, we present the drug effects on switch-related activity in the putamen for the high- and low-impulsive subjects separately. We observed that the effects of bromocriptine depended on trait impulsivity, as indicated by a significant group $\times$ drug interaction (bilateral putamen, $F_{(1,20)}=6.5, p=0.02$; the right putamen, $F_{(1,20)}=7.6, p=0.01$; the left putamen, $F_{(1,20)}=2.6, p=$ 0.1 ; bilateral caudate nucleus, $F_{(1,20)}=0.5$ ) (Fig. 5). Bromocriptine modulated switch-related activity in the right putamen of high-impulsive subjects $\left(F_{(1,9)}=11.9 ; p=0.007\right)$ but did not affect switch-related activity in the right putamen of lowimpulsive subjects $\left(F_{(1,11)}=0.2\right)$. Simple effects analyses revealed that the high-impulsive subjects on bromocriptine exhibited significantly greater activity in the right putamen on switch trials than on nonswitch trials $\left(F_{(1,9)}=7.2 ; p=0.03\right)$. Conversely, when they were on placebo, there was a trend toward greater right putamen activity on nonswitch trials than on switch trials $\left(F_{(1,9)}=3.9 ; p=0.08\right)$.

To assess this drug effect further, we analyzed whether the effect on switch-related activity in the right putamen was attrib-

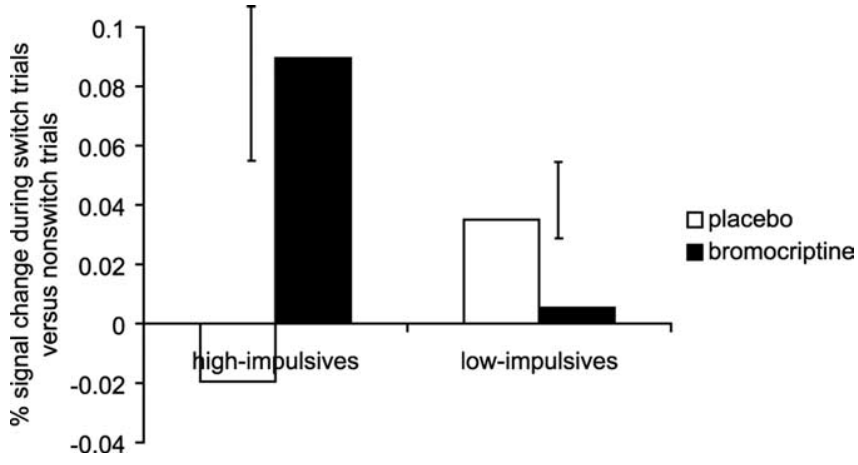

Figure 4. Signal change during switch trials minus nonswitch trials in the putamen. Data represent mean percentage signal change and are collapsed across the right and left putamen. Error bars represent SEs of the difference between treatment sessions.

utable to modulation of encoding-related activity during switch trials or nonswitch trials. Bromocriptine significantly attenuated activity on nonswitch trials $\left(F_{(1,9)}=12.5 ; p=0.006\right)$, whereas it left unaltered activity on switch trials $\left(F_{(1,9)}=0.2\right)$ (Table 3$)$. It may be noted that these latter simple main effects of bromocriptine are difficult to interpret given likely global or vascular effects that may differ between distinct brain regions (Krimer et al., 1998). We emphasize that drug effects on fMRI contrasts between comparable trial types (e.g., those between encoding on switch trials and encoding on nonswitch trials, as reported above) are more meaningful than simple main drug effects, because the former but not the latter control for global drug effects.

\section{Neural effects in the lateral frontal cortex}

We performed a second set of hypothesis-driven ROI analyses to test whether the drug effect on striatal activity during switching differed from that on lateral frontal activity during distraction. We conducted a repeated-measures ANOVA with group as the between-subjects factor and ROI (striatum vs lateral frontal cortex; see Materials and Methods for definition), process (switching vs distraction), and drug (bromocriptine vs placebo) as withinsubject factors. This ANOVA revealed that the drug differentially affected the striatum and the lateral frontal cortex during switching and distraction respectively, as evidenced by a significant $\mathrm{ROI} \times$ process $\times$ drug interaction $\left(F_{(1,20)}=4.4 ; p=0.05\right)$. Additional analyses revealed that the ROI $\times$ process $\times$ drug interaction was significant only in the high-impulsive subjects $\left(F_{(1,9)}=8.0 ; p=0.02\right.$ ) (Fig. 6) but not in the low-impulsive subjects $\left(\right.$ ROI $\times$ process $\times$ drug, $\left.F_{(1,11)}=0.1\right)$. There were no other effects of drug in the low-impulsive subjects, and we did not analyze these data further (main effect of drug, $F_{(1,11)}=0.3$; process $\times$ drug, $F_{(1,11)}=0.02$; ROI $\times$ drug, $\left.F_{(1,11)}=1.6\right)$.

To further analyze the drug effects on activity in the lateral 


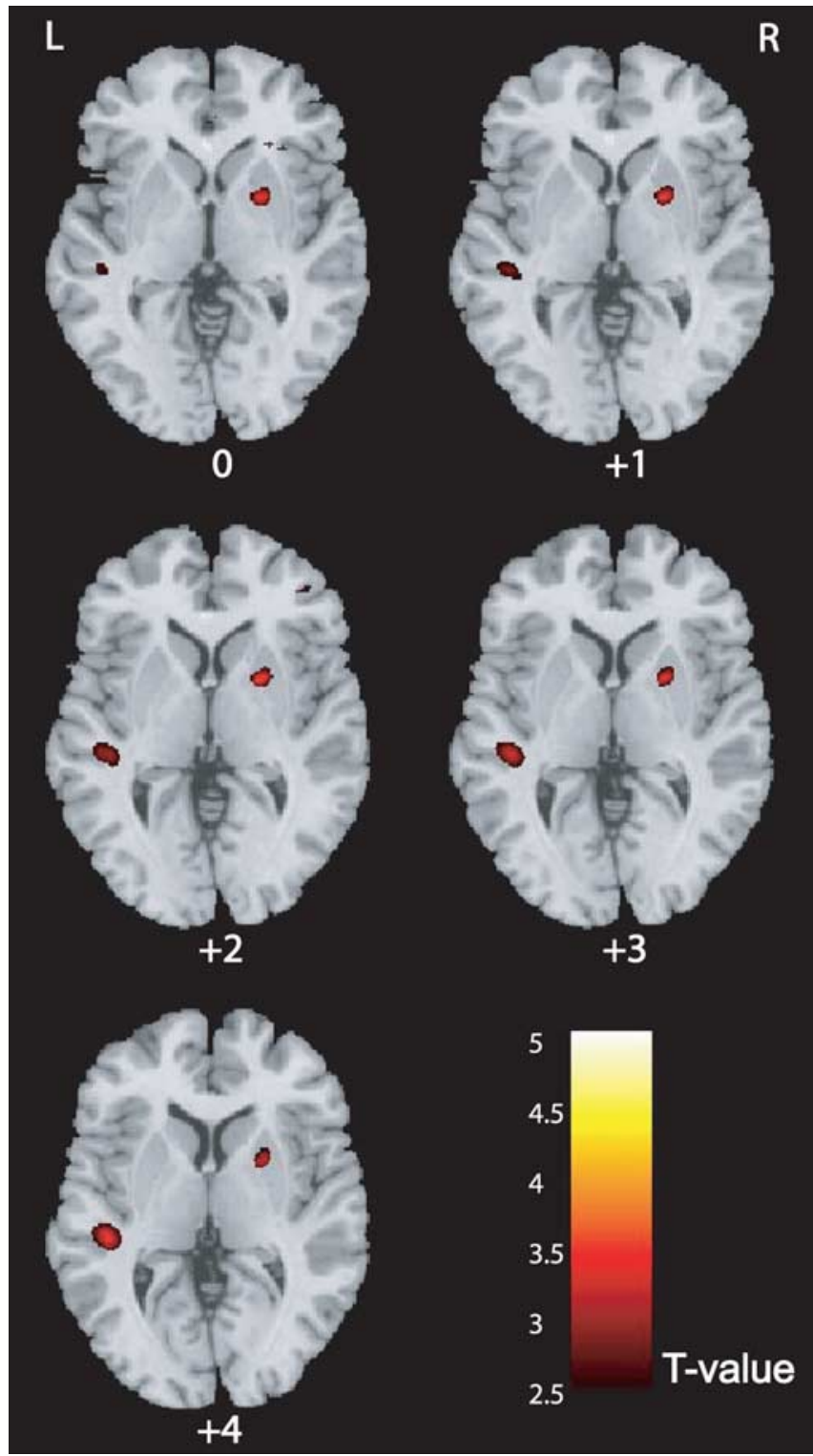

Figure 5. Effect of bromocriptine during switching as a function of trait impulsivity. Statistical parametric map superimposed on five axial sections [numbers below sections represent MNI z-coordinates (millimeters from the anterior commissure)] from the MNI template brain (the average of 27 scans from 1 subject to create the image known as "colin27") for the group $\times$ drug $X$ switch interaction contrast. A significant peak was observed in the right putamen [at coordinates $24,4,2(x, y, z)$; all $t$ values $>2.5$ are shown]. L, Left; $\mathrm{R}$, right.

Table 3. Encoding- and distractor-related percentage signal change from the right putamen

\begin{tabular}{llr}
\hline & Placeb0 & Bromocriptine \\
\hline High-impulsive & & \\
$\quad$ Nonswitch & $0.13(0.05)$ & $-0.02(0.05)$ \\
Switch & $0.06(0.05)$ & $0.08(0.04)$ \\
$\quad$ Nonscrambled distractor & $0.11(0.07)$ & $0.06(0.05)$ \\
Scrambled distractor & $0.09(0.07)$ & $0.04(0.04)$ \\
Low-impulsive & & \\
$\quad$ Nonswitch & $0.10(0.05)$ & $0.10(0.04)$ \\
Switch & $0.11(0.05)$ & $0.07(0.04)$ \\
Nonscrambled distractor & $0.07(0.04)$ & $0.005(0.03)$ \\
Scrambled distractor & $0.04(0.04)$ & $-0.01(0.05)$ \\
\hline
\end{tabular}

Values represent mean percentage signal change; values in parentheses represent SEM.
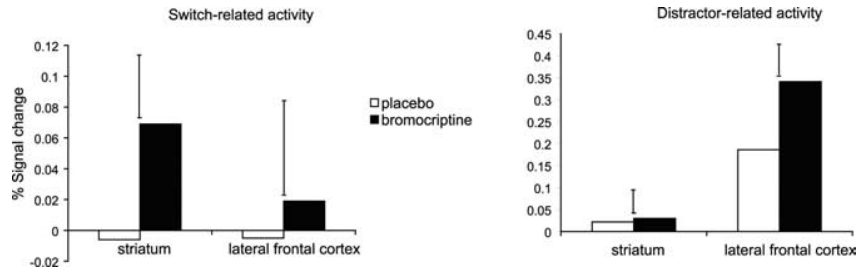

Figure 6. Signal change as a function of process, drug, and region of interest. Data represent mean percentage signal change in the striatum (collapsed across the four subregions) and the lateral frontal cortex (collapsed across the three subregions) for the high-impulsive subjects. Error bars represent SEs of the difference between drug sessions.

frontal cortex, we conducted additional simple effects analyses of the data from the high-impulsive subjects. These analyses revealed a significant drug effect on distractor-related activity in the lateral frontal cortex $\left(F_{(1,9)}=5.1 ; p=0.05\right)$ (Fig. 7) but not in the striatum $\left(F_{(1,9)}=0.02\right)$. Conversely, the drug did not affect activity in the lateral frontal cortex during switching $\left(F_{(1,9)}=0.3\right)$. Thus, bromocriptine modulated activity in the lateral frontal cortex during distraction, whereas it modulated the putamen during switching (see above). It may be noted that the drug effect on switch-related activity in the larger striatal meta-ROI only tended toward significance $\left(F_{(1,9)}=3.4 ; p=0.1\right)$, suggesting that the effects were specific to the putamen.

Next, we analyzed the spatial specificity of the drug effects in the lateral frontal cortex by looking at each sub-ROI separately in the high-impulsive subjects. The drug effect on distractor-related activity was attributable to effects on the left IFG $\left(F_{(1,9)}=5.9 ; p=\right.$ $0.04)$, in which the drug potentiated activity during the nonscrambled distractor $\left(F_{(1,9)}=8.0 ; p=0.02\right)$, while not affecting activity during the scrambled distractor $\left(F_{(1,9)}=1.4\right)$ (Table 4$)$. There were no effects on distractor-related activity in the right $\operatorname{IFG}\left(F_{(1,9)}=1.8\right)$ or the left MFG $\left(F_{(1,9)}=2.0\right)$.

Supplementary analyses confirmed that there were no effects on switch-related activity in the lateral frontal cortex, even when the larger meta-ROI was broken down into sub-ROIs [the left $\operatorname{IFG}\left(F_{(1,9)}=1.4\right)$, the right IFG $\left(F_{(1,9)}=0.5\right)$, or the left MFG $\left.\left(F_{(1,9)}=0.005\right)\right]$. Similar ANOVAs showed that there were no effects on distractor-related activity in the right putamen $\left(F_{(1,9)}=\right.$ $0.001)$, left putamen $\left(F_{(1,9)}=1.5\right)$, right caudate nucleus $\left(F_{(1,9)}=\right.$ $0.005)$, or left caudate nucleus $\left(F_{(1,9)}=1.8\right)$.

Finally, whole-brain analyses confirmed a significant drug $X$ group effect on switch-related activity in the striatum [activity peak centered on the right putamen at Talairach coordinates 24 , $4,2(x, y, z) ; p<0.001]$ but not the lateral frontal cortex. Conversely, there was a drug $\times$ group effect on distractor-related activity in the lateral frontal cortex [activity peak centered on the inferior frontal junction (Brass et al., 2005) (i.e., the border of the left IFG and the left inferior PCG) at coordinates 46, 4, $28(x, y, z)$; $p<0.001$ ] but not the striatum (Figs. 5, 7).

There were no significant correlations between the druginduced change in performance and drug-induced changes in neural activity.

\section{Summary of main results}

Bromocriptine improved attentional switching in high- but not low-impulsive subjects. This drug-induced improvement was accompanied by a drug-induced modulation of activity in the putamen, whereas lateral frontal activity was unaltered as a function of switching. Conversely, lateral frontal activity but not striatal activity was modulated by bromocriptine during distraction. This finding that bromocriptine had doubly dissociable effects in 


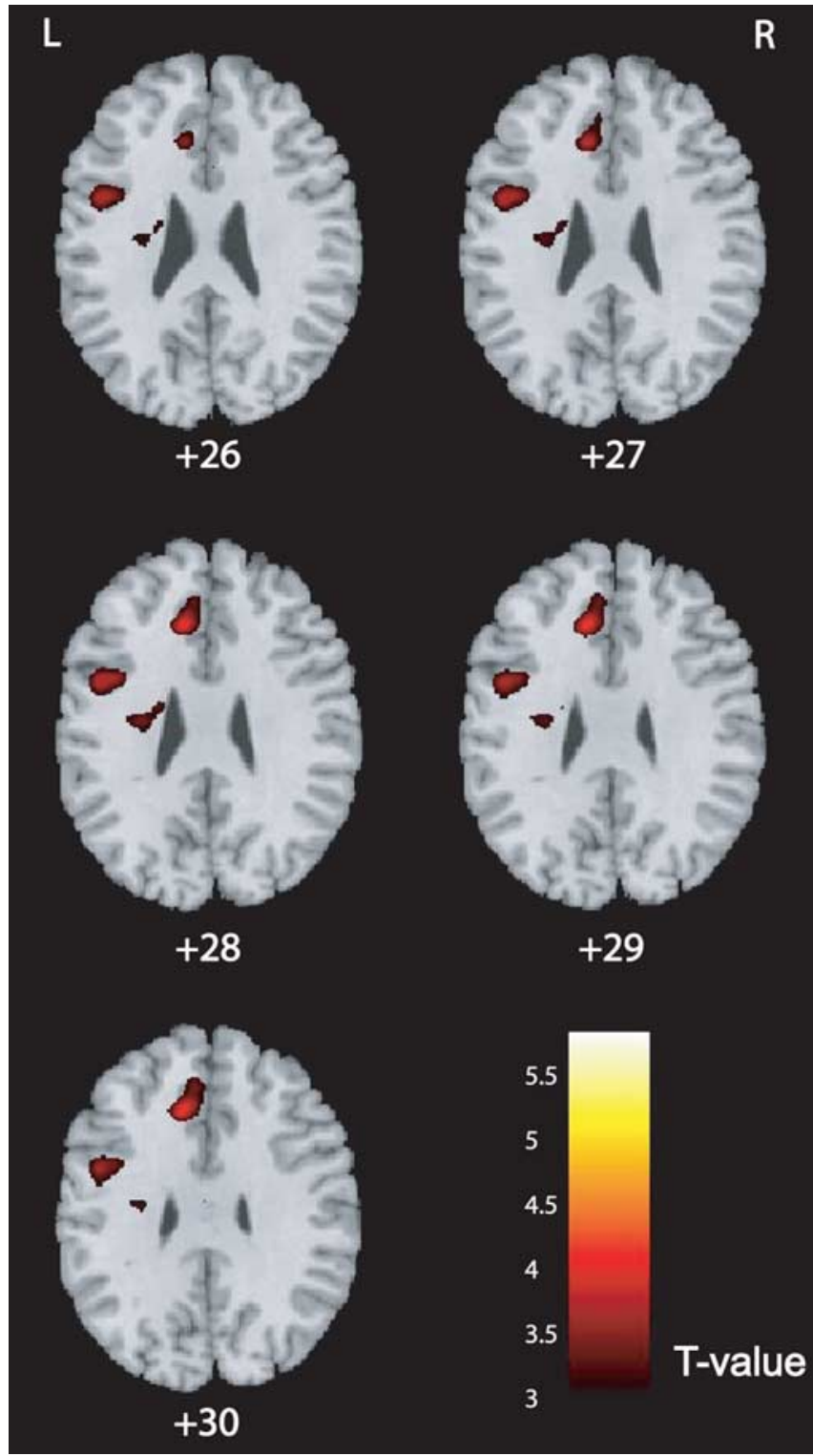

Figure 7. Effect of bromocriptine during distraction as a function of trait impulsivity. Statistical parametric map superimposed on five axial sections [numbers below sections represent MNI z-coordinates (millimeters from the anterior commissure)] from the MNI template brain (the average of 27 scans from 1 subject to create the image known as "colin27") for the group $X$ drug $X$ distractor interaction contrast. A significant peak was observed in the inferior frontal junction [i.e., the border of the left inferior frontal gyrus and left precentral gyrus; at coordinates $46,4,28(x, y, z) ;$ all $t$-values $>3.0$ are shown]. L, Left; $R$, right.

Table 4. Encoding- and distractor-related percentage signal change from the left IFG

\begin{tabular}{lll}
\hline & Placebo & Bromocriptine \\
\hline High-impulsive & & \\
$\quad$ Nonswitch & $0.21(0.07)$ & $0.19(0.06)$ \\
Switch & $0.21(0.07)$ & $0.18(0.06)$ \\
$\quad$ Nonscrambled distractor & $0.17(0.05)$ & $0.21(0.07)$ \\
$\quad$ Scrambled distractor & $0.19(0.06)$ & $0.20(0.06)$ \\
Low-impulsive & & \\
$\quad$ Nonswitch & $0.12(0.04)$ & $0.19(0.05)$ \\
Switch & $0.17(0.05)$ & $0.27(0.08)$ \\
Nonscrambled distractor & $0.27(0.08)$ & $0.32(0.09)$ \\
Scrambled distractor & $0.19(0.05)$ & $0.27(0.08)$ \\
\hline
\end{tabular}

Values represent mean percentage signal change; values in parentheses represent SEM. the striatum and the lateral frontal cortex as a function of trait impulsivity and component process was supported by both whole-brain and ROI analyses.

\section{Discussion}

We demonstrate that impulsive personality predicts DAdependent changes in frontostriatal activity during component processes of working memory. These results reveal the importance of taking into account individual variation in personality traits when predicting drug efficacy. In addition, they provide a key link between $\mathrm{D}_{2}$ receptor function, impulsivity, and frontostriatal activity during working memory.

The finding that trait impulsivity predicted effects on working memory concurs with previous studies showing that impulsecontrol disorders are accompanied not only by response control failures and abnormal reward processing (Jentsch and Taylor, 1999; Sonuga-Barke, 2002) but also by working memory impairment (Evenden, 1999; Schweitzer et al., 2000; Castellanos and Tannock, 2002; Willcutt et al., 2005). Indeed, a key characteristic of impulsive behavior is inappropriate attention to irrelevant information. DA dysfunction in frontostriatal systems may promote impulsivity by impairing the flexible updating of task relevance and inducing susceptibility to distraction in working memory (Frank et al., 2007).

We observed that bromocriptine improved switching, but only in high-impulsive subjects who had low baseline working memory capacity as measured with the reading span test. This finding is consistent with recent observations that the $\mathrm{D}_{2}$ receptor agonist cabergoline improved the flexible updating of information in working memory in healthy volunteers with low reading spans (Frank and O'Reilly, 2006). In addition, it concurs with results suggesting that dopaminergic medication improves the updating of information in working memory in patients with ADHD (Frank et al., 2007).

The present study revealed that the drug-induced improvement in switching was accompanied by a drug-induced modulation of putamen activity, again only in high-impulsive subjects. On placebo, poor performance on switch trials (relative to nonswitch trials) was accompanied by reduced putamen activity. This concurs with the hypothesis that striatal activity reflects the extent to which newly relevant information is gated into (i.e., allowed to update) working memory. Thus, on placebo, highimpulsive subjects were more proficient in updating working memory (and activating the striatum) on nonswitch trials than on switch trials. After bromocriptine, better performance on switch trials was accompanied by greater switch-related striatal activity. This is consistent with recent theorizing that DA opens the gate to working memory and renders the system more responsive to newly relevant inputs by acting at $\mathrm{D}_{2}$ receptors (Seamans and Yang, 2004) in the striatum (Frank et al., 2001). According to this account, bromocriptine attenuated the switch cost by allowing newly relevant information access to working memory.

Similar contrasting effects of DA have previously been observed as a function of individual genotypic variation in baseline DA. For example, the catechol O-methyltransferase $\mathrm{Val}^{158}-\mathrm{Met}$ genotype, which is known to be associated with variation in baseline DA, accounts for considerable variability in the effects of amphetamine (Mattay et al., 2003). Trait impulsivity has been associated with low baseline $\mathrm{DA} \mathrm{D}_{2} / \mathrm{D}_{3}$ receptor binding, as revealed by reduced uptake of the radioligand $\left[{ }^{18} \mathrm{~F}\right]$ fallypride in the striatum (Dalley et al., 2007). [ $\left.{ }^{18} \mathrm{~F}\right]$ Fallypride has high affinity for the $\mathrm{DA} \mathrm{D}_{2} / \mathrm{D}_{3}$ receptor and thus, reduced uptake of this radioli- 
gand likely indicates reduced dopamine $\mathrm{D}_{2} / \mathrm{D}_{3}$ receptor availability. The hypothesis that high-impulsive subjects exhibit low DA function is strengthened by the finding that, relative to lowimpulsive subjects, high-impulsive subjects exhibited significantly lower working memory capacity (reading span), which has been hypothesized to reflect baseline DA (Kimberg et al., 1997). Indeed contrasting effects of $\mathrm{DA}_{2}$ receptor agonists depending on reading span have previously been observed on switching and susceptibility to distraction (Kimberg et al., 1997; Frank and O’Reilly, 2006).

In keeping with previous suggestions (Frank et al., 2007), our findings suggest that effects on striatal activity during flexible updating in working memory may mediate the link between DA and impulsivity. The observation that the switch cost of high- and low-impulsive subjects did not significantly differ at baseline does not undermine this conclusion. It has been proposed that an “inverted U”-shaped relationship exists between DA and performance (Williams and Goldman-Rakic, 1995; Zahrt et al., 1997; Vijayraghavan et al., 2007), so that both too little and too much DA impairs performance. Our high- and low-impulsive subjects may be positioned on the left and right arm of the inverted $U$, respectively, at approximately similar distances from the optimum. This would account for both the opposite effects of bromocriptine and the lack of a significant difference at baseline.

Bromocriptine is an agonist, assumed to mimic DA, and its effects are frequently interpreted to reflect action at postsynaptic receptors. Our observation that bromocriptine potentiated motor tapping speed in both high- and low-impulsive subjects suggests that the drug acted postsynaptically in both groups, at least in systems underlying motor tapping. However, work with experimental animals has shown that low doses of bromocriptine act primarily at presynaptic $\mathrm{D}_{2}$ receptors, thereby paradoxically reducing DA release (Skirboll et al., 1979; Frank and O'Reilly, 2006). Therefore, it is possible that the beneficial effects of bromocriptine in high-impulsive subjects reflect a reduction of endogenous DA. This remains an outstanding issue to be addressed in future research.

It is recognized that the behavioral effects were modest. This is not surprising given that the effect reflected switching at encoding but was measured at probe. The significance of the behavioral effects is particularly meaningful given that they were accompanied by neural effects. The effects during switching were seen primarily in the putamen. This concurs with a recent study in which we observed that selective lesions in the putamen impaired cognitive switching (Cools et al., 2006) on a task that was previously shown to activate the putamen in healthy volunteers (Cools et al., 2004). Thus, the putamen plays a role in cognitive control as well as motor control.

The drug effects on striatal activity are unlikely to reflect nonspecific, global effects on the fMRI signal, because there was no effect during switching on task-responsive regions in the lateral frontal cortex. The null effects in the lateral frontal cortex do not reflect a lack of statistical power, because activity in the same region was significantly modulated during distraction. A role for the striatum in the dopaminergic modulation of flexibility concurs with recent theoretical work highlighting the importance of striatal DA in the flexible updating of currently active representations when change is required (Frank et al., 2001; Goto and Grace, 2005; Gruber et al., 2006). Furthermore, it concurs with a growing body of evidence for DA-dependent task-switching and working memory-updating deficits in patients with Parkinson's disease, which is characterized by striatal DA depletion (Cools et al., 2001a, 2006). The question of whether the effects observed here on the flexible updating of working memory extend to classic task switching is currently under investigation.

The finding that bromocriptine did not modulate the frontal cortex during switching is not to say that the frontal cortex does not contribute to flexibility. There is much evidence indicating a key role for the frontal cortex in task switching and set shifting (Dias et al., 1996; Sohn et al., 2000; Aron et al., 2004; Brass et al., 2005). However, DA may act at the level of the striatum to bias flexibility that itself depends on frontostriatal interactions. Indeed, Goto and Grace (2005) observed that injection of a $\mathrm{D}_{2}$ receptor agonist and antagonist into the striatum respectively attenuated and facilitated striatal responses that were invoked by stimulation of the frontal cortex. Moreover, they showed that injection of a $\mathrm{D}_{2}$ receptor agonist in the striatum of the rat impaired set shifting but only after inactivation of the (contralateral) frontal cortex. These data suggest that DA in the striatum serves attentional switching by mediating PFC-evoked input to the striatum.

Distractor-related activity was modulated by bromocriptine in the lateral frontal cortex and not in the striatum. This is consistent with the prediction that $\mathrm{D}_{2}$ receptor activation affects the impact of currently irrelevant inputs on the lateral frontal cortex (Seamans and Yang, 2004). Specifically, bromocriptine enhanced activity during the scrambled relative to the nonscrambled distractor in the lateral frontal cortex. The drug-induced increase in frontal activity may reflect enhanced recruitment of active maintenance processes to overcome distractibility, which concurs with the previous observation that DA-enhancing medication reduced susceptibility to distractors in patients with impulsive disorder (ADHD) (Frank et al., 2007). Alternatively, the activity increase may reflect greater impact of distractors and thus enhanced distractor susceptibility. Such enhanced distractibility was previously reported after administration of cabergoline in subjects with low reading span (Frank and O'Reilly, 2006). It is difficult to distinguish between these alternatives, because, despite the druginduced changes in the lateral frontal cortex, we did not observe a drug-induced change in the behavioral distractor cost. Indeed, the lack of distractor cost on placebo is a weakness of this study and suggests that the task was not sufficiently sensitive to detect behavioral distractibility, possibly because subjects did not emphasize speed during responding at probe. We recognize that the modulation of lateral frontal activity during distraction may reflect processes other than maintenance in the face of distraction, such as response inhibition. This does not undermine the main conclusion that the striatum and frontal cortex mediate the dopaminergic modulation of distinct component processes of working memory.

\section{References}

Arnsten AFT (1998) Catecholamine modulation of prefrontal cortical cognitive function. Trends Cogn Sci 2:436-446.

Aron A, Monsell S, Sahakian B, Robbins T (2004) A componential analysis of task-switching deficits associated with lesions of left and right frontal cortex. Brain 127:1561-1573

Beck AT, Ward CH, Mendelson M, Mock J, Erbaugh J (1961) An inventory for measuring depression. Arch Gen Psychiatry 11:561-571.

Benton AL (1968) Differential behavioral effects in frontal lobe disease. Neuropsychologia 6:53-60.

Boileau I, Dagher A, Leyton M, Gunn RN, Baker GB, Diksic M, Benkelfat C (2006) Modeling sensitization to stimulants in humans: an [11C]raclopride/positron emission tomography study in healthy men. Arch Gen Psychiatry 63:1386-1395.

Brass M, Derrfuss J, Forstmann B, Von Cramon D (2005) The role of the inferior frontal junction in cognitive control. Trends Cogn Sci 9:314-316. Brett M, Anton J-L, Valabregue R, Poline J-B (2002). Region of interest anal- 
ysis using an SPM toolbox. Paper presented at the 8th International Conference on Functional Mapping of the Human Brain, Sendai, Japan.

Brozoski TJ, Brown R, Rosvold HE, Goldman PS (1979) Cognitive deficit caused by regional depletion of dopamine in the prefrontal cortex of rhesus monkeys. Science 205:929-931.

Castellanos FX, Tannock R (2002) Neuroscience of attention-deficit/hyperactivity disorder: the search for endophenotypes. Nat Neurosci Rev 3:617-628.

Cools R (2006) Dopaminergic modulation of cognitive functionimplications for L-DOPA treatment in Parkinson's disease. Neurosci Biobehav Rev 30:1-23.

Cools R, Robbins TW (2004) Chemistry of the adaptive mind. Philos Transact A Math Phys Eng Sci 362:2871-2888.

Cools R, Barker RA, Sahakian BJ, Robbins TW (2001a) Mechanisms of cognitive set flexibility in Parkinson's disease. Brain 124:2503-2512.

Cools R, Barker RA, Sahakian BJ, Robbins TW (2001b) Enhanced or impaired cognitive function in Parkinson's disease as a function of dopaminergic medication and task demands. Cereb Cortex 11:1136-1143.

Cools R, Barker RA, Sahakian BJ, Robbins TW (2003) L-Dopa medication remediates cognitive inflexibility, but increases impulsivity in patients with Parkinson's disease. Neuropsychologia 41:1431-1441.

Cools R, Clark L, Robbins TW (2004) Differential responses in human striatum and prefrontal cortex to changes in object and rule relevance. J Neurosci 24:1129-1135.

Cools R, Ivry R, D'Esposito M (2006) The human striatum is necessary for responding to changes in stimulus relevance. J Cogn Neurosci 18: 1959-1972.

Crofts HS, Dalley JW, Van Denderen JCM, Everitt BJ, Robbins TW, Roberts AC (2001) Differential effects of 6-OHDA lesions of the frontal cortex and caudate nucleus on the ability to acquire an attentional set. Cereb Cortex 11:1015-1026.

Dalley JW, Fryer TD, Birchard L, Robinson ES, Theobald DE, Laane K, Pena Y, Murphy E, Shah Y, Probst K, Abakumova I, Aigbirho F, Richards HK, Hong Y, Baron JC, Everitt BJ, Robbins TW (2007) Nucleus accumbens D2/3 receptors predict trait impulsivity and cocaine reinforcement. Science 315:1267-1270.

Daneman M, Carpenter P (1980) Individual differences in working memory and reading. J Verbal Learn Verbal Behav 19:450-466.

Dawe S, Loxton NJ (2004) The role of impulsivity in the development of substance use and eating disorders. Neurosci Biobehav Rev 28:343-351.

Dias R, Robbins TW, Roberts AC (1996) Dissociation in prefrontal cortex of affective and attentional shifts. Nature 380:69-72.

Durstewitz D, Seamans J, Sejnowski T (2000) Dopamine-mediated stabilization of delay-period activity in a network model of prefrontal cortex. J Neurophysiol 83:1733-1750.

Evenden J (1999) Varieties of impulsivity. Psychopharmacology 146: $348-361$.

Frank M, Loughry B, O’Reilly R (2001) Interactions between frontal cortex and basal ganglia in working memory: a computational model. Cogn Affect Behav Neurosci 1:137-160.

Frank M, Santamaria A, O'Reilly R, Willcutt E (2007) Testing computational models of dopamine and noradrenaline dysfunction in attention deficit/hyperactivity disorder. Neuropsychopharmacology, in press.

Frank MJ, O'Reilly RC (2006) A mechanistic account of striatal dopamine function in human cognition: psychopharmacological studies with cabergoline and haloperidol. Behav Neurosci 120:497-517.

Gibbs SE, D'Esposito M (2005a) Individual capacity differences predict working memory performance and prefrontal activity following dopamine receptor stimulation. Cogn Affect Behav Neurosci 5:212-221.

Gibbs SE, D'Esposito M (2005b) A functional MRI study of the effects of bromocriptine, a dopamine receptor agonist, on component processes of working memory. Psychopharmacology (Berl) 180:644-653.

Goto Y, Grace A (2005) Dopaminergic modulation of limbic and cortical drive of nucleus accumbens in goal-directed behavior. Nat Neurosci 8:805-812.

Grant DA, Berg EA (1948) A behavioural analysis of degree of reinforcement and ease of shifting to new responses in a Weigl-type card sorting problem. J Exp Psychol 38:404-411.

Gruber AJ, Dayan P, Gutkin BS, Solla SA (2006) Dopamine modulation in the basal ganglia locks the gate to working memory. J Comput Neurosci 20:153-166.
Jentsch J, Taylor J (1999) Impulsivity resulting from fronto-striatal dysfunction in drug abuse: implications for the control of behavior by reward-related stimuli. Psychopharmacology 146:373-390.

Keilp JG, Sackeim HA, Mann JJ (2005) Correlates of trait impulsiveness in performance measures and neuropsychological tests. Psychiatry Res 135:191-201.

Kimberg DY, D’Esposito M, Farah MJ (1997) Effects of bromocriptine on human subjects depend on working memory capacity. NeuroReport 8:3581-3585.

Krimer L, Muly E, Willimas G, Goldman-Rakic P (1998) Dopaminergic regulation of cerebral cortical microcirculation. Nat Neurosci 1:286-289.

Mattay V, Goldberg T, Fera F, Hariri A, Tessitore A, Egan M, Kolachana B, Callicot J, Weinberger D (2003) Catechol O-methyltransferase Val ${ }^{158}$ -met genotype and individual variation in the brain response to amphetamine. Proc Natl Acad Sci USA 100:6186-6191.

Milner B (1971) Interhemispheric differences and psychological processes in man. Br Med Bull 27:272-277.

Nasreddine Z, Phillips N, Bédirian V, Charbonneau S, Whitehead V, Collin I, Cummings J, Chertkow H (2005) The Montreal Cognitive Assessment (MoCA): a brief screening tool for mild cognitive impairment. J Am Geriatr Soc 53:695-699.

Nelson HE (1982) National Adult Reading Test (NART) test manual. Windsor, UK: NFER-Nelson.

Owen AM, Herrod NJ, Menon DK, Clark JC, Downey SPMJ, Carpenter A, Minhas PS, Turkheimer FE, Williams EJ, Robbins TW, Sahakian BJ, Petrides M, Pickard D (1999) Redefining the functional organization of working memory processes within human lateral prefrontal cortex. Eur J Neurosci 11:567-574.

Patton J, Stanford M, Barratt E (1995) Factor structure of the Barratt impulsiveness scale. J Clin Psychol 51:768-774.

Phillips A, Ahn S, Floresco S (2004) Magnitude of dopamine release in medial prefrontal cortex predicts accuracy of memory on a delayed response task. J Neurosci 14:547-553.

Sawaguchi T, Goldman-Rakic PS (1991) D1 dopamine receptors in prefrontal cortex: involvement in working memory. Science 251:947-950.

Schweitzer JB, Faber TL, Grafton ST, Tune LE, Hoffman JM, Kilts CD (2000) Alterations in the functional anatomy of working memory in adult attention deficit hyperactivity disorder. Am J Psychiatry 157:278-280.

Seamans JK, Yang CR (2004) The principal features and mechanisms of dopamine modulation in the prefrontal cortex. Prog Neurobiol 74:1-58.

Skirboll L, Grace A, Bunney B (1979) Dopamine auto- and postsynaptic receptors: electrophysiological evidence for differential sensitivity to dopamine agonists. Science 206:80-82.

Smith S (2002) Fast robust automated brain extraction. Hum Brain Mapp 17:143-155.

Sohn M, Ursu S, Anderson JR, Stenger VA, Carter CS (2000) The role of prefrontal cortex and posterior parietal cortex in task switching. Proc Natl Acad Sci USA 97:13448-13453.

Sonuga-Barke EJ (2002) Psychological heterogeneity in AD/HD-a dual pathway model of behaviour and cognition. Behav Brain Res 130:29-36.

Stroop J (1935) Studies of interference in serial verbal reactions. J Exp Psychol 18:643-662.

Tzourio-Mazoyer N, Landeau B, Papathanassiou D, Crivello F, Etard O, Delcroix N, Mazoyer B, Joliot M (2002) Automated anatomical labeling of activations in SPM using a macroscopic anatomical parcellation of the MNI MRI single-subject brain. NeuroImage 15:273-289.

Vijayraghavan A, Wang M, Birnbaum SG, Williams GV, Arnsten AFT (2007) Inverted-U dopamine D1 receptor actions on prefrontal neurons engaged in working memory. Nat Neurosci 10:376-384.

Willcutt E, Doyle A, Nigg J, Faraone S, Pennington B (2005) Validity of the executive function theory of attention-deficit/hyperactivity disorder: a meta-analytic review. Biol Psychiatry 57:1336-1346.

Williams GV, Goldman-Rakic PS (1995) Blockade of dopamine D1 receptors enhance memory fields of prefrontal neurons in primate cerebral cortex. Nature 376:572-575.

Yoon JH, Curtis CE, D'Esposito M (2006) Differential effects of distraction during working memory on delay-period activity in the prefrontal cortex and the visual association cortex. NeuroImage 29:1117-1126.

Zahrt J, Taylor JR, Mathew RG, Arnsten AFT (1997) Supranormal stimulation of $\mathrm{D}_{1}$ dopamine receptors in the rodent prefrontal cortex impairs spatial working memory performance. J Neurosci 17:8528-8535. 